/04/ 


\section{RURAL BLENDED EDUCATION. PROYECTO DE EDUCACIÓN SEMIPRESENCIAL PARA FRENAR LA DESPOBLACIÓN DE LAS ZONAS RURALES}

RURAL BLENDED EDUCATION. BLENDED EDUCATIONALPROJECTTOREDUCEDEPOPULATION IN RURAL AREAS

Fulgencio Sánchez Giménez

Docente Formación Profesional.

Masterado en Formación del Profesorado. Universidad Antonio de Nebrija.

Masterado e-Learning y Tecnología Educativa. Universidad Camilo José Cela. Masterado en Gestión y Dirección de Centros Educativos. Universidad CEU-UCH.

Doctorando Ciencias Sociales. Universidad Católica de Murcia (España). E-mail: sanchez_fulgim@gva.es ORCID: https://orcid.org/0000-0003-1491-1537

Recepción: 07/12/2018. Aceptación: 17/01/2019. Publicación: 29/03/2019

Gitación sugerida:

Sánchez Giménez, F. (2019). Rural Blended Education. Proyecto de educación semipresencial para frenar la despoblación de las zonas rurales. 3C TIC. Cuadernos de desarrollo aplicados a las TIC, 8(1), pp. 74-95. doi:http:// dx.doi.org/10.17993/3ctic.2019.81.74-95 


\section{RESUMEN}

El objetivo de este artículo es presentar un proyecto de innovación denominado "Rural Blended Education" que demuestre que implementado un nuevo paradigma educativo basado en un sistema de enseñanza semipresencial en un Colegio de Educación Infantil y Primaria se puede revertir el fenómeno de la despoblación en una zona geográfica. El estudio comienza analizando las causas y las consecuencias de la despoblación y qué se está haciendo para frenarla. Una vez el estado de la cuestión ha sido presentado, se formula una hipótesis de trabajo que considera la posibilidad de frenar esta tendencia mediante el desarrollo de un proceso de enseñanzaaprendizaje semipresencial en estas zonas. El estudio se implementa como un diseño de un proyecto de intervención contextualizado en la población de las aldeas diseminadas de la comarca de UtielRequena (Valencia). En este diseño se confecciona un proyecto de innovación en el que se forma al profesorado para mejorar su competencia digital, para después crear una serie de herramientas con las que padres y madres puedan educar cooperativamente a sus hijos e hijas. De igual manera, se temporaliza el proyecto, se establecen las líneas de trabajo del mismo, se determinan los requisitos para participar en el programa, se realiza una memoria económica del mismo y se trazan una serie de indicadores para determinar si el sistema ha sido viable o no. Por último, se presentan unas conclusiones al proyecto, así como las limitaciones y recomendaciones más importantes que han de seguirse en posteriores cursos académicos.

\section{ABSTRACT}

The aim of this article is to present an innovation project called "Rural Blended Education" that demonstrates that depopulation in a given geographical area can be reduced by applying a new educational paradigm in a School of Early Childhood and Primary Education. The study starts with an analysis of the the causes and consequences of depopulation and the actions taken to slow down this trend. Then, we formulate a working hypothesis that attempts to consider if depopulation can be stopped by improving the channels in the teaching-learning process of these areas. The study is implemented as an intervention project design carried out in the villages scattered across the Utiel-Requena area 
(Valencia). A key aspect of this innovation project is to train teachers in their digital competence first, and then create a series of cooperative tools with which parents can co-educate their children. In the same way, the timing of the project and the main lines of work are established, the requirements to participate in the program are set out, an economic report is made and a series of indicators are presented to determine if the system has been viable or not. Finally, conclusions are drawn, together with the most important limitations and recommendations to consider in subsequent academic courses.

\section{PALABRAS CLAVE}

Despoblación, Educación cooperativa, Campus virtual, Competencia digital, Formación semipresencial.

\section{KEYWORDS}

Depopulation, Cooperative education, Learning Management System, Digital competence, Blended learning. 


\section{INTRODUCCIÓN}

La despoblación es un fenómeno que se extiende por casi toda Europa, especialmente en los países del Sur, como España. Afecta a un tercio de las regiones europeas y en muchas de ellas hoy llueve sobre mojado porque ya sufrieron una gran dispersión geográfica entre 1960 y 1970, que vació el campo español y concentró las poblaciones demográficas en las grandes ciudades. 70 años después vuelven a encenderse las alarmas con un diagnóstico claro, población envejecida, baja natalidad y éxodo de jóvenes hacia las áreas urbanas (Cabré y Pérez, 1995).

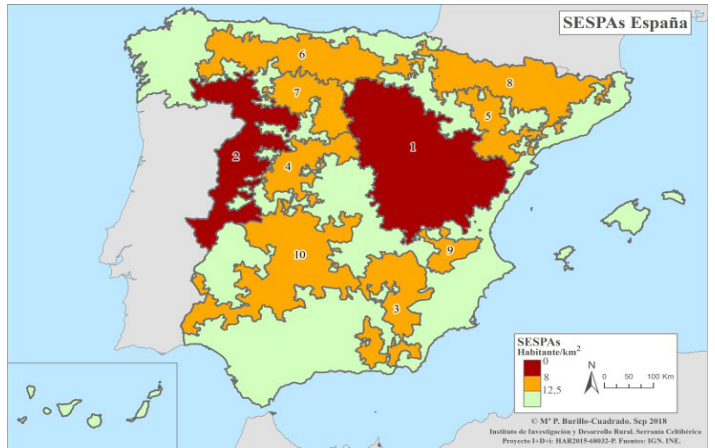

Figura 1. Mapa de la despoblación en España.

Fuente: Instituto de Desarrollo Rural Serranía Celtibérica (2018).

\begin{tabular}{|c|c|c|c|c|}
\hline \multicolumn{5}{|c|}{ SESPAs de España } \\
\hline SESPAs (2017) & № Municipios & Población & Area $\left(\mathrm{km}^{2}\right)$ & Densidad (hab $/ \mathrm{km}^{2}$ ) \\
\hline 1. Serranía Celtibérica & 1.383 & 498.086 & 69.162 & 7,20 \\
\hline 2. Franja con Portugal & 586 & 253.690 & 33.477 & 7,58 \\
\hline 3. Serranía Bética & 169 & 192.228 & 21.495 & 8,94 \\
\hline 4. Serranía Central & 477 & 139.258 & 13.979 & 9,96 \\
\hline 5. Tierras del Ebro & 174 & 123.440 & 12.275 & 10,06 \\
\hline 6. Serranía Cantábrica & 378 & 298.329 & 29.526 & 10,10 \\
\hline 7. Tierras del Duero & 400 & 152.538 & 13.914 & 10,96 \\
\hline 8. Pirineos & 477 & 328.998 & 29.559 & 11,13 \\
\hline 9. Serranía Levantina & 50 & 64.285 & 5.715 & 11,25 \\
\hline 10. Sierra Morena y Submeseta & 281 & 502.562 & 43.893 & 11,45 \\
\hline Total & 4.375 & 2.553 .414 & 272.995 & 9,35 \\
\hline Resto de España & 3.749 & 44.018 .718 & 232.996 & 188,92 \\
\hline
\end{tabular}

Figura 2. Tabla analítica de la despoblación en España.

Fuente: Instituto de Desarrollo Rural Serranía Celtibérica (2018). 
Se ha de decir que el fenómeno de la despoblación afecta a más del 50\% por ciento de la población española (concretamente el 53\% de ésta), donde tan sólo se recoge apenas dos millones y medio de personas. Datos estos que aportan una densidad poblacional de 9,35 hab/km2, por debajo de la cifra de 10 hab/km2 que en Geografia Humana se denomina "desierto demográfico" (Burillo, 2018).

\section{DESARROLLO}

\subsection{REQUISITOS PARA FRENAR LA DESPOBLACIÓN}

Para conseguir revertir esta fuga poblacional, se deben aunar esfuerzos por parte de todas las administraciones, con tal de conseguir favorecer cinco factores fundamentales que se insinúan imprescindibles para repoblar las zonas desfavorecidas (Herráiz Hernansanz y Serrano Gil, 2013):

1. Un acceso asequible a una vivienda digna.

2. Facilidad para acceder a un empleo, con posibilidades del desarrollo de autoempleo o acceso a internet con calidad para poder trabajar el teletrabajo.

3. Presencia de una serie de servicios mínimos de abastecimiento alimenticio y de energía.

4. Acceso a sanidad rural.

5. Acceso a la educación tanto Primaria como Secundaria.

En esta tarea, las Administraciones Públicas tienen la misión de luchar activamente contra esta realidad, ya que no es posible, que más del 50\% del territorio nacional cuente únicamente con el 5\% de la población. A continuación se proponen las principales acciones que éstas están desarrollando:

a. Unión Europea: La Unión Europea está realizando grandes esfuerzos en todos los ámbitos de actuación, estudiando los desafíos demográficos a los que ha de enfrentarse Europa, así como las recomendaciones que deberían de llevar a cabo sus estados miembros ante esta situación (Comité Europeo de las Regiones, 2017). Pero además ha considerado dar 
prioridad a la recepción de fondos a todos los proyectos que tengan como finalidad atajar lo que el Parlamento Europeo llama "el Reto Demográfico" como se hace eco "La Tribuna de Salamanca" (2018).

b. Gobierno de España: Se están comenzando a promover ayudas concretas, como las que el pasado 5 de Octubre de 2018, aprobó el Consejo de Ministros, de 80 millones de euros, extraídos del Programa de Empleo Juvenil del Fondo Social Europeo para el periodo 20142020 para apoyar a los jóvenes entre 18 y 30 años que deseen emprender y/o favorecer el teletrabajo en zonas despobladas (Real Decreto 1234/2018). A pesar de que esta cifra resulta exigua para dar respuesta a un problema que afecta a más de la mitad territorio nacional, se ha de aplaudir esta primera medida tangible implementada por el Gobierno de España. Según recoge la Federación Española de Municipios y Provincias, FEMP (2018) la senda de directrices nacionales deberían establecer medidas más amplias que traten de realizar planes de empleo local y comarcal y propuestas de apoyo directo a autónomos.

c. Administraciones autonómicas: A modo de ejemplo, en la Comunidad Valenciana aún no se han materializado ayudas ni planes específicos, pero, se ha puesto en marcha una la Comisión para la Lucha contra la Despoblación (Decreto 58/2017) y se ha desarrollado un Programa de Desarrollo Rural para la Comunidad Valenciana de 2014 a 2020.

d. Ayuntamientos: Sería necesario estudiar medidas concretas de muchos ayuntamientos de nuestro país, pero se citará uno a modo de ejemplo. El Ayuntamiento de Medina de Rioseco realiza anualmente las Jornadas Autonómicas de la Confederación de Centros de Desarrollo Rural, como la celebrada el 28 de Septiembre de 2018 y donde el consistorio del mencionado pueblo ha recogido un plan contra la despoblación con 21 medidas llamado "Rioseco 21XXI" (Carpintero, 2018). 


\subsection{INTERNET RURAL}

Una de las principales acciones para frenar la despoblación es reducir la brecha digital, ofreciendo una conexión a Internet, que se extienda a más cantidad de áreas y con mayor calidad. ¿Pero cuál es la situación actual de la conectividad de Internet en zonas rurales? ¿Está llegando un Internet de calidad a todos los rincones de nuestro país? Si se toma con referencia empresas externas, para medir la cantidad y calidad de señal de Internet, se puede ver que la realidad es muy diferente a la que proponen las operadoras en sus campañas publicitarias. La empresa Open Signal ${ }^{\circledR}$ permite explorar mediante un mapa de calor la intensidad de 4G que llega a todas las zonas españolas comprobando que éste llega únicamente a los principales núcleos poblacionales. La importancia del 4G, en ambientes rurales es imprescindible para el desarrollo de la zona, el 4G facilita el autoempleo, el teletrabajo o el ocio en la red. En la Orden ECE/116/2018 (2018) el Estado obliga a las grandes operadoras de telefonía a que antes de que acabe el año 2020 las mencionadas multinacionales tengan que ofrecer un servicio de $30 \mathrm{Mbps}$ de descarga al 90\% de los habitantes de municipios menores de 5000 habitantes.

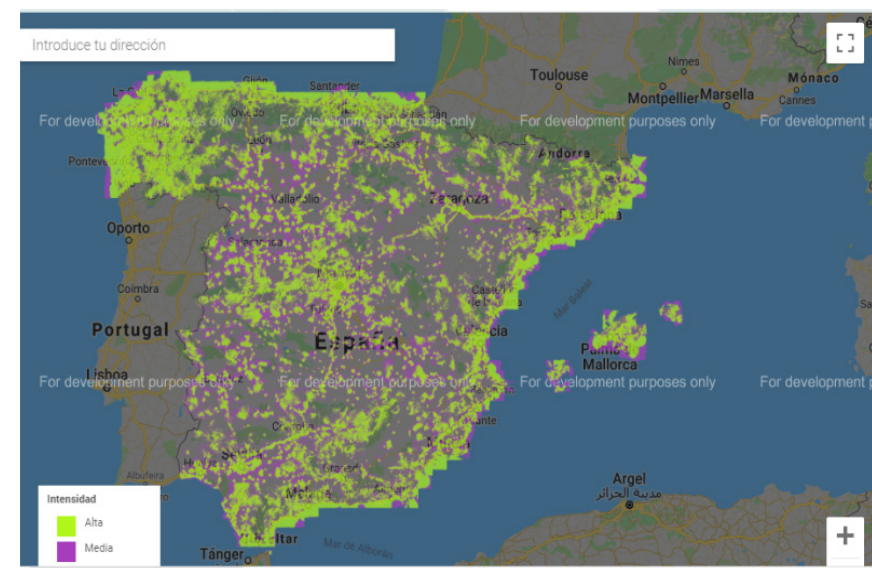

Figura 3. Conectividad de $4 \mathrm{G}$ en el territorio nacional.

Fuente: Open Signal (2018). 


\subsection{SITUACIÓN DE LA EDUCACIÓN EN LAS ZONAS RURALES}

La Educación Primaria en las zonas rurales está determinada por un tipo de centros llamados Colegios Rurales Agrupados, que se representan con el acrónimo GRA. Lacort, (2014) propone que una de las mayores carencias de una escuela rural es que no pueden contar con los medios que cuentan los colegios convencionales, pero a pesar de ser imposible abastecer a las escuelas rurales de estos medios materiales muchos pedagogos como Feu i Gelis, (2004) proponen que la formación en estos entornos ofrece numerosos beneficios para los alumnos y alumnas, que son benchmarking para el sistema educativo.

\subsection{LÍMITES DE LOS COLEGIOS RURALES AGRUPADOS}

En función de cada Comunidad Autónoma existe un mínimo de alumnos determinado para que un CRA no tenga que cerrar sus puertas. Una de las CCAA con la ratio más baja es Castilla y León y Aragón donde la ratio es de 4 alumnos (LaNuevaCrónica.com, 2018), aunque en el resto de Comunidades Autónomas estas ratios van aumentando, así por ejemplo es de cinco alumnos en La Rioja y en la Comunidad Valenciana, seis en Galicia u ocho en Cataluña (Cebrián, 2017). Guando el CRA tiene que cerrarse por la escasez de niños éstos deben salvar la distancia de su domicilio al centro educativo en autobús, son internos de lunes a viernes en la Escuela Rural o la familia se traslada a la localidad del centro educativo ahondando en el problema de la despoblación.

Cuando el CRA tiene que cerrarse por la escasez de niños éstos deben salvar la distancia de su domicilio al centro educativo en autobús, son internos de lunes a viernes en la Escuela Rural o la familia se traslada a la localidad del centro educativo ahondando en el problema de la despoblación.

Sería en este momento cuando se podría formular la hipótesis de trabajo a nuestra investigación ¡Se podrían implementar una formación académica basada en las TICs para formar a los alumnos cuando se tiene que cerrar un CRA? Para Cabero Almenara (2016), la inclusión de las nuevas tecnologías está ofreciendo nuevos sistemas de formación a distancia que permiten el acceso a los contenidos a personas que están lejos del lugar donde se encuentra el formador. Algunos de estos casos serían: 
- Personas que solapen otra actividad en el momento que se está produciendo la formación.

- Estudiantes de zonas rurales lejanos a los lugares de formación.

- Alumnados incomunicados por inclemencias meteorológicas durante periodos invernales.

- Discapacitados físicos que no puedan salvar las barreras arquitectónicas para acceder a la sede de la formación.

- Familias con cambio reiterado de la vivienda habitual.

- Niños y niñas de padres separados que viven lejos entre sí y que tienen custodia compartida.

- Personas privadas de libertad que deseen completar o complementar su formación.

\subsection{SITUACIÓN DE LA EDUCACIÓN PRIMARIA A DISTANCIA LEGISLATIVAMENTE}

El Ministerio de Educación ha creado el CIDEAD (Centro para la Innovación y Desarrollo de la Educación a Distancia) que desarrolla un sistema pedagógico y tecnológico para conseguir una educación a distancia diversificada (García, 2010) para las etapas de Educación Primaria, Secundaria y Bachillerato a personas que viven en el extranjero (Real Decreto 789/2015). Con lo cual se puede concretar que un sistema de formación e-learning para formar a estudiantes de Primaria ya se encuentra implementado y se podría extrapolar esta consideración a un entorno rural que dé amparo a su población con el fin de parar la despoblación.

\subsection{DESARROLLO DEL MODELO “RURAL BLENDED”}

Para desarrollar este modelo semipresencial es necesario llevar a cabo las acciones siguientes:

1. Presentar el proyecto en el Claustro de Profesores comprobando cuál es su grado de aceptación y presentando las ventajas del programa:

a. Ser pioneros en España de un proyecto educativo que trate de frenar la despoblación. 
b. Permitir compatibilizar la vida familiar con vida profesional al profesorado ya que sólo deberá asistir un día al Centro Educativo.

c. Posibilidad de extrapolar el proyecto a otras realidades:

- De manera provisional: familias itinerantes, discapacitados físicos temporales, hospitalizados, niños acosados o niños acosadores, hijos de padres divorciados con custodia compartida y con residencia lejanas una de la otra....

- De manera permanente: discapacitados físicos, alumnos que abandonan el país....

\section{Presentar el proyecto a los padres y madres como modelo opcional en el proceso de} enseñanza-aprendizaje de sus hijos remarcando los pros y contras del programa (Véase Tabla 1):

Tabla 1. Ventajas e inconvenientes del programa para las familias.

\begin{tabular}{|c|c|}
\hline PROS & CONTRAS \\
\hline $\begin{array}{l}\text { Los y las estudiantes evitan hacer largos desplazamientos } \\
\text { o estar internos durante la semana académica en una } \\
\text { residencia rural. } \\
\text { Se palia la dispersión geográfica de su aldea. } \\
\text { Se crea un sentimiento de arraígo a su población. } \\
\text { La educación cooperativa ofrece a padres y madres la opción } \\
\text { de la participación activa en el proceso educativo de sus hijos. } \\
\text { Es una educación más flexible en cuanto al tiempo y el } \\
\text { espacio. } \\
\text { Las sesiones quedan grabadas por lo que el acceso a los } \\
\text { materiales se puede realizar en cualquier momento y durante } \\
\text { sucesivas ocasiones. } \\
\text { Potencia la competencia digital de los padres y madres y de } \\
\text { los menores. } \\
\text { Favorece la formación inicial de todos los integrantes del } \\
\text { entorno familiar. } \\
\text { Esta medida reduce los desplazamientos en carretera y por } \\
\text { ende mejora el medio ambiente. }\end{array}$ & $\begin{array}{l}\text { Requiere que los padres formen a sus hijos a diario y } \\
\text { controlen su evolución. } \\
\text { Requiere desplazamientos una vez por semana al centro de } \\
\text { referencia por parte de los menores. } \\
\text { Se debe prestar atención atención para conseguir la máxima } \\
\text { socialización de los niños y las niñas tanto en la estancia en } \\
\text { el colegio como en la vida familiar. }\end{array}$ \\
\hline
\end{tabular}

Fuente: elaboración propia (2018). 
Tabla 2. Deberes y obligaciones de padres y madres y profesorado.

\section{PADRES Y MADRES}

Disponer de conexión a Internet en casa (como mínimo de 3G).

Asistir de manera presencial al curso de competencia digital para padres, donde se explicará el uso de la plataforma, debiéndose superar una prueba práctica que se realizará tras su conclusión

Asistir a una sesión virtual de 1 hora semanal donde se planificará la acción formativa doméstica. Asistir, a las sesiones presenciales de contenidos curriculares de formación para padres y madres, si los contenidos tratados, no son dominados por el progenitor.

Efectuar un seguimiento de la acción formativa que el menor realiza, alentándole en las actividades a realizar y ofreciéndole feed-back de los trabajos que realice.

Manejar, durante la acción formativa el ordenador que el centro facilite a la familia, ya que, en éste estarán instalados los programas que se utilizarán para la formación.

Responder los mails que el profesorado le envíe a padres y madres.

Usar a diario Google Calendar como herramienta planificativa de tareas.

\section{PROFESORADO}

Dominar la plataforma LMS, que permitirá subir temas, vídeos, actividades..

Grabar vídeos de los contenidos de estudio, ya sea de la propia sesión con el resto de alumnado presencial, mediante la realización de sesiones de Streaming, o Master-Class asíncrona con los contenidos más relevantes de cada bloque temático.

Subir el material didáctico al aula virtual.

Desarrollar la acción formativa inicial de competencia digital a padres/madres y alumnos.

Realizar una hora de formación virtual online con el padre o madre, dentro del horario lectivo del profesor,

con el fin de planificar la acción formativa de la semana, así cómo asesorar en las en las dificultades pedagógicas que el menor presente.

Ofrecer asistencia técnica a los padres mediante programas de control remoto en las sesiones de tutoria inidividual virtual.

Participar en la formación presencial de los padres.

Responder los mails que los padres y el menor le envíe.

Usar a diario Google Calendar como herramienta de planificación de tareas.

Fuente: elaboración propia (2018).

3. Evaluación de la competencia digital del profesorado: En esta evaluación se comprobará cuál es el punto de partida de la formación del profesorado, por medio de un cuestionario básico sobre su competencia digital. 
4. Verificación de la adecuación del alumnado en el proyecto a desarrollar: Será el/la psicólogo/a del Centro mediante una entrevista el que determine si el niño/a reúne las características adecuadas para sumarse al programa.

\section{Redactar y firmar un contrato bilateral entre padres y madres y profesorado}

con las exigencias de la formación doméstica y de la formación escolar. Dicho contrato presentará las condicionantes que ambas partes han de cumplir (Ver Tabla 2).

6. Previsión de costes del programa y previsión de costes sin programa: Dentro de la presentación del Proyecto se recomendaría reflejar una previsión de costes si el programa no se implementara (Ver Tabla 3).

Tabla 3. Costes de implementación programa y costes si éste no se llevara a cabo.

\begin{tabular}{|c|c|c|c|c|}
\hline RECURSO & \multicolumn{2}{|c|}{ IMPLEMENTACION PROGRAMA } & \multicolumn{2}{|c|}{ NO IMPLEMENTACIÓN PROGRAMA } \\
\hline \multirow{3}{*}{$\begin{array}{l}\text { Recursos } \\
\text { humanos }\end{array}$} & $\begin{array}{c}2 \text { profesores que gestionan los } 24 \\
\text { alumnos }\end{array}$ & 47.500 & $\begin{array}{l}1 \text { profesor que gestiona } \\
\text { los } 24 \text { alumnos }\end{array}$ & 23.750 \\
\hline & 1 Experto en TICs para curso & \multirow{2}{*}{1.800} & 3 Servicios autobús diario & 66.000 \\
\hline & formación inicial profesorado & & Limpieza del aula & 1300 \\
\hline \multirow{2}{*}{$\begin{array}{l}\text { Recursos } \\
\text { materiales }\end{array}$} & $\begin{array}{c}\text { Amortización } 24 \text { ordenadores } \\
\text { portátiles }\end{array}$ & 2.400 & Calefacción & 800 \\
\hline & Amortización 24 Impresoras & 720 & $\begin{array}{c}\text { Amortización material } \\
\text { Centro }\end{array}$ & 1000 \\
\hline \multirow{3}{*}{$\begin{array}{l}\text { Recursos } \\
\text { tecnicos }\end{array}$} & Móvil empresa profesor & 460 & & \\
\hline & $\begin{array}{l}\text { Conexión a Internet para } \\
\text { profesorado y alumnado }\end{array}$ & 11.040 & & \\
\hline & $\begin{array}{l}\text { Licencias en el programa } \\
\text { informáticos }\end{array}$ & 750 & & \\
\hline TOTAL & & 64670 & & 92.850 \\
\hline
\end{tabular}

Fuente: elaboración propia (2018).

7. Planificación de la acción formativa: Una de las mayores dificultades de la implementación de este modelo Semipresencial reside, en los desconocimientos técnicos que este modelo formativo suele ofrecer al profesorado en el momento de comenzar a implementarlo. Es por ello que se debe estructurar un curso de formación dedicado a la exposición al profesorado de herramientas necesarias para realizar una formación desde entornos virtuales, con garantías de calidad a los padres y madres y a los escolares. En este 
curso de formación se trabajará con la plataforma Schoology, y se implementará formación sobre herramientas útiles para creación de entornos B-learning:

- Grabación de vídeo y pantalla de ordenador (Screencast-o-Matic).

- Edición de vídeo (Movie Maker).

- Gestión de videos (canal de YouTube).

- Uso de plataforma de comunicación con videoconferencia (Hangouts).

- Aplicaciones útiles TICs para el trabajo en entornos virtuales (Kahoot, Edpuzzle, Mindomo, Tagxedo...).

- Gestión remoto de equipos (Team Viewer)....

\section{Evaluación. Trazo de indicadores para medir la calidad del proyecto: Para} comprobar si realmente se están cumpliendo los resultados esperados en el programa es preciso trazar una serie de indicadores que permitan determinar si éste está siendo viable (Ver Tabla 4). Cada curso académico se evaluará 4 veces los siguientes indicadores para estudiar la viabilidad del programa (Septiembre, Enero, Abril y Junio).

9. Calendario semanal durante el desarrollo del proyecto: Es conveniente delimitar cuáles son las funciones de cada uno de los Stackeholders, dentro de la realización del programa. Para ello sería recomendable, marcar las horas presenciales y a distancia de cada uno de los involucrados en el programa. Se exponen a modo de ejemplo los horarios semanales de cada uno de ellos (Ver Tablas 5, 6, 7 y 8). 
Tabla 4. Indicadores utilizados en el proyecto "Rural Blended Education".

\begin{tabular}{|c|c|c|c|}
\hline & PADRES/MADRES & ALUMNOSIAS & PROFESORADO \\
\hline Indicador subjetivo & \multicolumn{3}{|c|}{$\begin{array}{l}\text { Grado de satisfacción del proyecto (escala de Likert). } \\
\text { Motivación en el desarrollo del proyecto (escala de Likert). }\end{array}$} \\
\hline Indicador objetivo & $\begin{array}{l}\text { Tiempo dedicado al proceso } \\
\text { formativo del hijo/a. }\end{array}$ & $\begin{array}{c}\text { Resultados académicos } \\
\text { obtenidos (con respecto a } \\
\text { otros años). } \\
\text { Resultados académicos } \\
\text { obtenidos con respecto } \\
\text { a compañeros/as de su } \\
\text { mismo curso. }\end{array}$ & $\begin{array}{l}\text { Tiempo de trabajo semanal } \\
\text { (una vez desarrollado los } \\
\text { contenidos). }\end{array}$ \\
\hline
\end{tabular}

Fuente: elaboración propia (2018).

Tabla 5. Calendario semanal de Stakeholders en el proyecto "Rural Blended Education".

\begin{tabular}{|c|c|c|}
\hline & LUNES A JUEVES (ONLINE) & VIERNES (PRESENCIAL) \\
\hline \multirow{3}{*}{ Padres /Madres } & 4 horas de docencia & $\begin{array}{l}4 \text { horas presenciales en Centro de formación en } \\
\text { contenidos curriculares específicos (Optativas) }\end{array}$ \\
\hline & 4 horas de apoyo & \multirow{2}{*}{$\begin{array}{l}2 \text { horas presenciales de Socialización en Centro } \\
\text { (Optativas) }\end{array}$} \\
\hline & 1 hora de tutoría individual con tutor & \\
\hline \multirow{3}{*}{ Alumnos/as } & \multirow{3}{*}{$\begin{array}{l}\text { 16-20 horas trabajo en casa (docencia } \\
\text { paterna, trabajo LMS, resolución tareas...) }\end{array}$} & $\begin{array}{c}4 \text { horas de Asignaturas de Música y Educación Física } \\
\text { (Obligatorias) }\end{array}$ \\
\hline & & $\begin{array}{c}2 \text { horas presenciales de Socialización en Centro } \\
\text { (Optativas) }\end{array}$ \\
\hline & & $\begin{array}{l}3 \text { horas dedicadas a actividades cooperativas, } \\
\text { resolución dudas y pruebas escritas. }\end{array}$ \\
\hline Profesor/a & $\begin{array}{c}12 \text { horas para asistencia a padres } \\
\text { (Obligatorias) } \\
3 \text { horas de resolución remoto de } \\
\text { problemas (Obligatorias) } \\
6 \text { horas de creación de contenidos }\end{array}$ & $\begin{array}{c}2 \text { horas de asistencia a Claustros } \\
4 \text { horas de formación a padres } \\
3 \text { horas formación/apoyo o evaluación niños/as }\end{array}$ \\
\hline
\end{tabular}

Fuente: elaboración propia. 
Tabla 6. Ejemplo de calendario docente de los familiares en el proyecto "Rural Blended Education".

\begin{tabular}{|c|c|c|c|c|c|}
\hline \multicolumn{6}{|c|}{ CALENDARIO DOCENTE PADRE/MADRE } \\
\hline & $\begin{array}{l}\text { Lunes } \\
\text { (Online) }\end{array}$ & $\begin{array}{c}\text { Martes } \\
\text { (Online) }\end{array}$ & $\begin{array}{c}\text { Miércoles } \\
\text { (Online) }\end{array}$ & $\begin{array}{l}\text { Jueves } \\
\text { (Online) }\end{array}$ & $\begin{array}{c}\text { Viernes } \\
\text { (Presenciales) }\end{array}$ \\
\hline \multicolumn{6}{|l|}{8.00 a 9.00} \\
\hline \multicolumn{6}{|l|}{9.00 a 10.00} \\
\hline 10.00 a 11.00 & \multicolumn{4}{|c|}{ Horas apoyo docente al menor (Obligatorias) } & \multirow{3}{*}{$\begin{array}{c}\text { Formación en } \\
\text { contenidos } \\
\text { curriculares } \\
\text { (Optativas) }\end{array}$} \\
\hline 11.00 a 12.00 & $\begin{array}{c}\text { Tutoría } \\
\text { Individual } \\
\text { Tutor Centro } \\
\text { Educativo }\end{array}$ & & & & \\
\hline \multicolumn{5}{|l|}{12.00 a 13.00} & \\
\hline 13.00 a 14.00 & \multicolumn{4}{|c|}{ Horas resolución dudas al menor (Obligatorias) } & Actividades \\
\hline 14.00 a 15.00 & & & & & $\begin{array}{l}\text { socialización } \\
\text { (Optativas) }\end{array}$ \\
\hline
\end{tabular}

Fuente: elaboración propia (2018).

Tabla 7. Ejemplo de calendario formación al menor en el proyecto "Rural Blended Education".

\begin{tabular}{|c|c|c|c|c|c|}
\hline \multicolumn{6}{|c|}{ CALENDARIO FORMACIÓN MENOR } \\
\hline & $\begin{array}{l}\text { Lunes } \\
\text { (Online) }\end{array}$ & $\begin{array}{l}\text { Martes } \\
\text { (Online) }\end{array}$ & $\begin{array}{l}\text { Miércoles } \\
\text { (Online) }\end{array}$ & $\begin{array}{l}\text { Jueves } \\
\text { (Online) }\end{array}$ & $\begin{array}{c}\text { Viernes } \\
\text { (Presenciales) }\end{array}$ \\
\hline 8.00 a 9.00 & & & & & \\
\hline 9.00 a 10.00 & & & & & \\
\hline 10.00 a 11.00 & \multicolumn{4}{|c|}{ Horas apoyo docente al menor (Obligatorias) } & Música y Ed. Física \\
\hline 11.00 a 12.00 & \multirow{2}{*}{\multicolumn{4}{|c|}{ Horas de trabajo autónomo del menor }} & (Obligatoria) \\
\hline 12.00 a 13.00 & & & & & \\
\hline 13.00 a 14.00 & \multicolumn{4}{|c|}{ Horas resolución dudas al menor (Obligatorias) } & Actividades \\
\hline 14.00 a 15.00 & & & & & $\begin{array}{l}\text { socialización } \\
\text { (Obligatorias) }\end{array}$ \\
\hline 15,00 a 16.00 & & & & & Realización \\
\hline 16.00 a 17.00 & & & & & actividades \\
\hline 17.00 a 18.00 & & & & & $\begin{array}{c}\text { cooperativas, } \\
\text { resolución dudas y } \\
\text { pruebas escritas. } \\
\text { (Obligatorias) }\end{array}$ \\
\hline
\end{tabular}

Fuente: elaboración propia (2018). 
Tabla 8. Ejemplo de calendario docente para el profesor/a del Centro Educativo en el proyecto "Rural Blended Education".

\begin{tabular}{|c|c|c|c|c|c|}
\hline \multicolumn{6}{|c|}{ CALENDARIO DOCENTE PROFESOR/A } \\
\hline & $\begin{array}{l}\text { Lunes } \\
\text { (Online) }\end{array}$ & $\begin{array}{l}\text { Martes } \\
\text { (Online) }\end{array}$ & $\begin{array}{l}\text { Miércoles } \\
\text { (Online) }\end{array}$ & $\begin{array}{l}\text { Jueves } \\
\text { (Online) }\end{array}$ & $\begin{array}{c}\text { Viernes } \\
\text { (Presenciales) }\end{array}$ \\
\hline 8.00 a 9.00 & & & & & \\
\hline 9.00 a 10.00 & $\begin{array}{c}\text { Tutoria } \\
\text { Pad/Mad } 10\end{array}$ & \multirow{2}{*}{\multicolumn{2}{|c|}{$\begin{array}{l}\text { Horas de creación de } \\
\text { contenidos }\end{array}$}} & $\begin{array}{c}\text { Tutoria } \\
\text { Pad/Mad } 12\end{array}$ & \multirow{4}{*}{$\begin{array}{c}\text { Formación en } \\
\text { contenidos } \\
\text { curriculares } \\
\text { (Obligatorias) }\end{array}$} \\
\hline 10.00 a 11.00 & $\begin{array}{c}\text { Tutoria } \\
\text { Pad/Mad } 3\end{array}$ & & & & \\
\hline 11.00 a 12.00 & & $\begin{array}{c}\text { Tutoria } \\
\text { Pad/Mad } 1\end{array}$ & $\begin{array}{c}\text { Tutoria } \\
\text { Pad/Mad } 11\end{array}$ & $\begin{array}{c}\text { Tutoria } \\
\text { Pad/Mad } 7\end{array}$ & \\
\hline 12.00 a 13.00 & & \multicolumn{3}{|c|}{ Horas de asistencia remota } & \\
\hline 13.00 a 14.00 & & & & & \multirow{2}{*}{ Claustros } \\
\hline 14.00 a 15.00 & & & & & \\
\hline 15,00 a 16.00 & & & & & \multirow{4}{*}{$\begin{array}{l}\text { Realización } \\
\text { actividades } \\
\text { cooperativas, } \\
\text { resolución dudas y } \\
\text { pruebas escritas. } \\
\text { (Obligatorias) }\end{array}$} \\
\hline 16.00 a 17.00 & $\begin{array}{c}\text { Tutoria } \\
\text { Pad/Mad } 8\end{array}$ & $\begin{array}{c}\text { Tutoria } \\
\text { Pad/Mad } 2\end{array}$ & $\begin{array}{c}\text { Tutoria } \\
\text { Pad/Mad } 9\end{array}$ & $\begin{array}{c}\text { Tutoria } \\
\text { Pad/Mad } 4\end{array}$ & \\
\hline 17.00 a 18.00 & $\begin{array}{c}\text { Tutoria } \\
\text { Pad/Mad } 5\end{array}$ & $\begin{array}{c}\text { Tutoria } \\
\text { Pad/Mad } 6\end{array}$ & \multicolumn{2}{|c|}{$\begin{array}{l}\text { Horas de creación de } \\
\text { contenidos }\end{array}$} & \\
\hline 18.00 a 19.00 & & & & & \\
\hline 19.00 a 20.00 & & \multicolumn{3}{|c|}{ Horas de asistencia remota } & \\
\hline 20.00 a 21.00 & & & & & \\
\hline
\end{tabular}

Fuente: elaboración propia (2018).

10. Memoria: Presentación de resultados $\mathrm{Al}$ final de cada año, con el fin de controlar que el proyecto se está implementando se debería de evaluar:

A. Evolución de indicadores medios subjetivos: Satisfacción con el programa y motivación para continuar en el mismo, a lo largo del curso, para cada uno de los Stakeholders.

B. Horas de trabajo medio diario para cada uno de los Stakeholders durante el periodo del trimestre.

C. Resultados académicos obtenidos por los estudiantes target del programa en relación a los resultados obtenidos por un grupo control.

D. Causas de abandono del programa.

E. Previsión de recursos económicos, materiales y humanos en función de las necesidades formativas para el curso académico siguiente. 


\section{CONCLUSIONES}

Equiparar las oportunidades, derechos y servicios de los pueblos a las ciudades es la estrategia para que el medio rural no se convierta en un enorme espacio vacío para el turismo de fin de semana. Estas actuaciones, según estudios contrastados, deben incidir sobre cinco pilares sobre los que se asienta el éxodo rural: la escasez de viviendas en medio rural, la falta de empleo en estas zonas, la lejanía a los servicios de primera necesidad, la pérdida de los servicios de atención primaria de salud en la localidad y la disminución de los centros educativos de Primaria y Secundaria cercanos a los núcleos rurales.

Un sistema basado en la Educación Semipresencial, donde padres y madres y profesores participen activamente con la educación de sus hijos, podría ser un sistema para atajar el último factor que se ha mencionado. Este sistema no es nuevo, ya que lo está desarrollando el Ministerio de Educación Cultura y Deporte para estudiantes que viven en el extranjero a través de su Centro de Innovación y Desarrollo, denominado CIDEAD.

Las actuaciones mencionadas, según estudios contrastados, deben incidir sobre cinco pilares sobre los que se asienta el éxodo rural

Además, el sistema no sólo serviría para atajar la despoblación, sino que podría implementarse en otros colectivos: familias itinerantes, niños discapacitados físicos, niños acosados o alumnos que tengan el rol de acosadores, padres divorciados con custodia compartida y con residencia lejanas una de la otra...

No obstante, para poder desarrollar el programa, éste tendría que ser aceptado por cada uno de los Stakeholders (profesorado, familias, alumnado y administraciones educativas), además de llevar implícito un sistema de formación en competencia digital al profesorado y a las familias, así como un control riguroso de la planificación temporal, unas obligaciones de enseñanza del profesorado 
y de las familias aprendizaje de alumnos y, por último se deben trazar unos indicadores claros, concisos y mensurables que permitan comprobar si éste está cumpliendo las expectativas del mismo.

\section{REFERENCIAS BIBLIOGRÁFICAS}

Burillo, P. (2018). La investigadora Pilar Burillo cartografí la despoblación de España por agrupación de municipios como recomienda la Comisión Europea. La Serranía Celtibérica. Recuperado el 4 de Noviembre de 2018 de: http:/ / www.celtiberica.es/despoblacion-y-cambios-demograficos-los-retos-de-la-politica-europea/

Cabré, A. y Pérez, J. (1995). Envejecimiento demográfico en España. Recuperado de: http:/ / digital.csic. es/bitstream/10261/38745/1/1995seniors.pdf

Cabero Almenara, J. (2016). La educación a distancia como estrategia de inclusión social y educativa. Revista Mexicana de Bachillerato a Distancia, 8(15).

Carpintero, D. (2018, 12 de febrero). Un Plan contra la despoblación con cien medidas en tres años. Lavozderioseco.com.

Cebrián, M. (2017, 27 de septiembre). Colegios con 3 alumnos: la supervivencia de la escuela rural en Aragón. Canales de Vice.

Decreto 58/2017, por el que se crea la Comisión Interdepartamental para la Lucha contra el Despoblamiento de los Municipios Valencianos, Documento Oficial de la Comunitat Valenciana, de 28 de abril de 2017.

Dictamen del Comité Europeo de las Regiones 2017/C 017/08, de 17 de enero, de la respuesta de la UE al reto demográfico. Diario Oficial de la Unión Europea, de 18 de enero de 2017.

García, J. J. (2010) La Educación a Distancia en los distintos sistemas educativos de la Unión Europea. Recuperado de: https://m.monografias.com/trabajos31/educacion-distancia-sistemaseducativos-europa/educacion-distancia-sistemas-educativos-europa.shtml

Herráiz, J. y Serrano, O. (2013). Despoblación y abandono de las actividades agropecuarias tradicionales en la Serranía Alta de Cuenca: medidas de desarrollo sostenible en un territorio rural desfavorecido (Tesis doctoral) 
Universidad de Granada, Granada. Recuperado de: http://hdl.handle.net/10481/27525

FEMP. (2017). Documento de acción. Comisión de la despoblación. Listado de medidas para luchar contra la despoblación. Recuperado el 18 de noviembre de 2018 de: http:/ / www.femp.es/sites/default/ files/multimedia/documento_de_accion_comision_de_despoblacion_9-05-17.pdf

Feu i Gelis, J. (2004). La escuela rural en España: apuntes sobre las potencialidades pedagógicas, relacionales y humanas de la misma Revista Digital eRural, Educación, cultura y desarrollo rural, 2(3). Recuperado de: http://educación.upa.cl/revistaerural/erural.htm

Instituto de Desarrollo Rural Serranía Celtibérica. (2018). Situación de despoblación en España. [Gráfico]. Recuperado el 12 de noviembre de 2018 de: http://www.celtiberica.es/wp-content/ uploads/2018/10/SESPA_Esp_rec-1024x695.jpg

Lacort Navarro, J. (2014) La identidad del alumnado en la práctica docente diaria actual en un Colegio Rural Agrupado. (Trabajo Fin de Grado). Universidad Internacional de la Rioja. Recuperado de: https:// reunir.unir.net/bitstream/handle/123456789/2569/lacort.navarro.pdf ?sequence=1

OpenSignal (2018). Gestión de la señal móvil de Internet. [Gráfico] Recuperado de: https:/ /opensignal. com/

Orden EGE/1166/2018, de 29 de octubre, por la que se aprueba el Plan para proporcionar cobertura que permita el acceso a servicios de banda ancha a velocidad de 30 Mbps o superior, a ejecutar por los operadores titulares de concesiones en la banda de 800 Mhz. Boletín Oficial del Estado, de 8 de noviembre de 2018.

Real Decreto 789/2015, de 4 de septiembre, por el que se regula la estructura y funcionamiento del Centro para la Innovación y el Desarrollo de la Educación a Distancia. Boletín Oficial del Estado, de 23 de septiembre de 2015.

Real Decreto 1234/2018, de 5 de octubre, por el que se establecen las bases reguladoras para la concesión directa de subvenciones a entidades locales para la financiación de proyectos de empleo, autoempleo y emprendimiento colectivo, dirigidos a afrontar el reto demográfico en los municipios de menor población, en el marco del Programa 
3C TIC. Cuadernos de desarrollo aplicados a las TIC. ISSN: 2254-6529

Operativo de Empleo Fuvenil del Fondo Social Europeo (ayudas EMP-POEJ). Boletín Oficial del Estado, de 6 octubre de 2018.

Tribuna Salamanca. (2018, 16 de octubre). La despoblación, criterio para fondos europeos y priorizar proyectos. TribunaSalamanca. Recuperado de: https://www.tribunasalamanca.com/ noticias/la-despoblacion-criterio-para-recibir-fondos-europeos-y-priorizar-proyectos/ 1539723929 


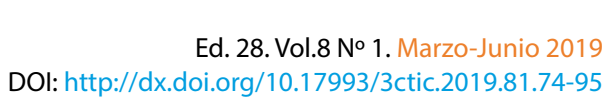

